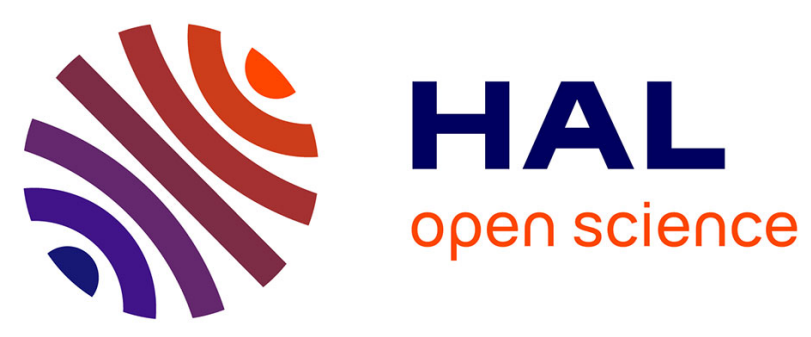

\title{
Ultrafast diagnostics
}

J.-C. Diels, J.J. Fontaine, W. Rudolph

\section{To cite this version:}

J.-C. Diels, J.J. Fontaine, W. Rudolph. Ultrafast diagnostics. Revue de Physique Appliquée, 1987, 22 (12), pp.1605-1611. 10.1051/rphysap:0198700220120160500 . jpa-00245718

\section{HAL Id: jpa-00245718 https://hal.science/jpa-00245718}

Submitted on 1 Jan 1987

HAL is a multi-disciplinary open access archive for the deposit and dissemination of scientific research documents, whether they are published or not. The documents may come from teaching and research institutions in France or abroad, or from public or private research centers.
L'archive ouverte pluridisciplinaire HAL, est destinée au dépôt et à la diffusion de documents scientifiques de niveau recherche, publiés ou non, émanant des établissements d'enseignement et de recherche français ou étrangers, des laboratoires publics ou privés. 


\title{
IMPULSIONS ULTRACOURTES
}

\author{
Classification \\ Physics Abstracts \\ $42.60 \mathrm{H}$
}

\section{Ultrafast diagnostics}

\author{
J.-C. Diels, J. J. Fontaine $(*)$ and W. Rudolph $(* *)$ \\ Department of Physics and Astronomy, The University of New Mexico, Albuquerque, New Mexico 87131, \\ U.S.A.
}

(Reçu le 9 juin 1987, accepté le 21 juillet 1987)

\begin{abstract}
Résumé. - Les techniques de corrélation du second ordre permettent une caractérisation complète, en amplitude et en phase, des impulsions ultrabrèves. Nous présentons quelques applications de ces techniques, en particulier une méthode d'imagerie tridimensionnelle en second harmonique où des informations peuvent être obtenues sur l'objet par balayage en profondeur uniquement.
\end{abstract}

\begin{abstract}
Second order correlation techniques can be used to obtain a complete characterization of femtosecond pulses, in amplitude and phase. The importance of monitoring and controlling the frequency content of ultrashort pulses is discussed. Application of correlation techniques - in particular to three dimensional diagnostics - are reviewed. It is shown that depth scanning alone is sufficient to generate three dimensional images by up-conversion range gating.
\end{abstract}

\section{Introduction.}

Recent progress in the generation of ultrashort pulses have opened the way to a large number of new applications in diverse fields as physics, electronics, chemistry, and biology. The purpose of this paper is to review recent progress in spatial and temporal correlation techniques, which make it possible to achieve the optimal temporal and spatial resolution in any particular experiment. New techniques to determine the pulse shape in amplitude and phase are discussed in section 2. Accurate pulse characterization is not a luxury, but a real need for a large variety of temporal as well as spatial applications (Sect. 3). Knowledge of the pulse shape is obviously important when the temporal resolution is marginal, and deconvolution techniques have to be used in order to extract the pulse rise time. Knowledge and control of the pulse phase modulation makes distortion compensation possible, both in temporal and spatial applications. We will show in particular that, with a particular choice of cavity configuration, precompensation of a femtosecond pulse can be achieved, such that a minimum pulse

(*) Present address : ENSAIS, Strasbourg, France.

$(* *)$ Present address : Friedrich-Schiller Universitat, 6700 Jena, GDR. duration is obtained at the desired location of an experimental set-up (at the end of an amplifier chain, or at the location of a target in imaging applications). A new and powerful application of ultrashort optical correlation techniques to three dimensional imaging is presented in section 4.

\section{Pulse characterization.}

Correlation techniques are needed whenever electronic techniques fail to provide acceptable temporal resolution. There is no absolute limit of the time scale where correlation techniques have to take over more « direct » method. Rather, every field has its particular limits. For instance, the nanosecond time domain should be considered as ultrashort in high voltage and high current applications. This is the first domain of application for which deconvolution techniques are developed [1, 2]. Picosecond pulses deserve the "ultrashort " designation at the $\mathrm{CO}_{2}$ wavelength, since they represent only a few optical cycles, while in optics, it is only in the femtosecond time scale that correlation techniques become essential. How can one determine exactly the temporal characteristics of a fs light pulse, given that there is no faster pulse to probe it with? The traditional answer to this problem has been the standard second order auto-correlation discussed below. 
2.1 The BASIC CORRElator. - An example of typical second order autocorrelator is sketched in figure 1 , where a linearly polarized beam is split into two orthogonally polarized equal parts by a polarizing beam splitter cube. Each beam is sent through a
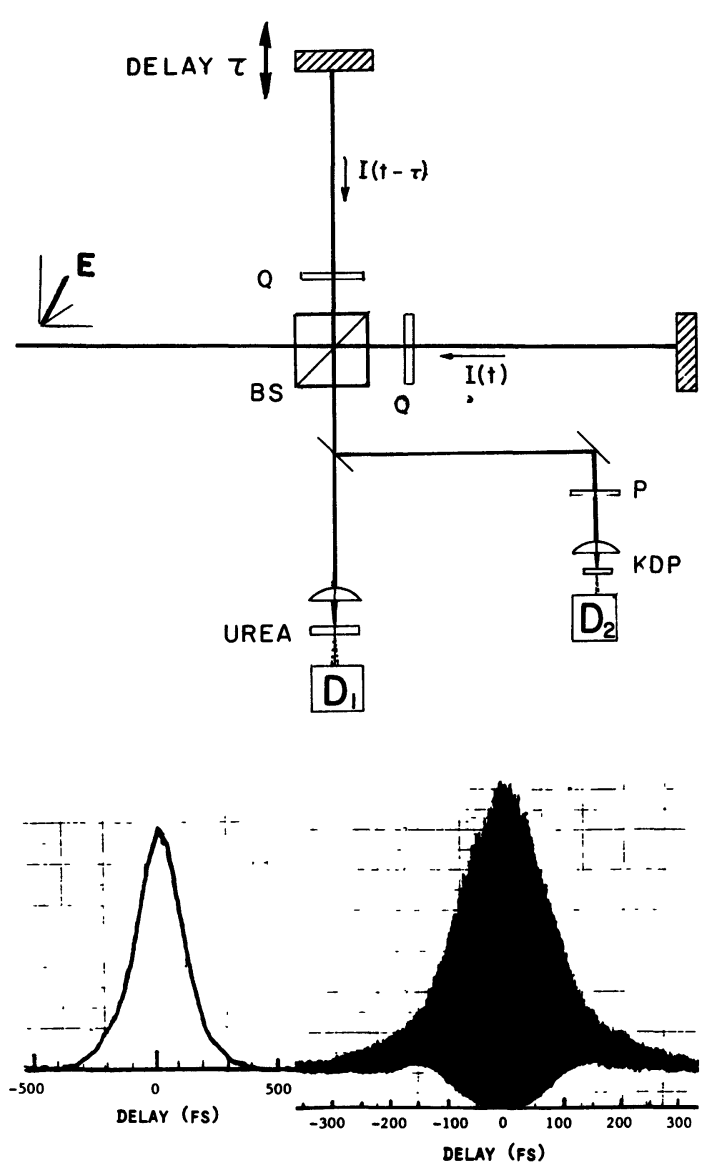

Fig. 1. - The basic auto-correlator. The beam carrying the pulse train is polarized at $45^{\circ}$ to the plane of the figure, to be split into two equal parts by the polarizing beam splitter BS. With a quarter wave plate $Q$ inserted in each arm of the correlator, the beams reflected by the fixed and movable (delay $\tau$ ) mirrors are sent, orthogonally polarized, into a urea crystal. This nonlinear crystal is oriented for phase matched second harmonic generation type II. The signal recorded by the detector $D_{1}$ is thus proportional to the intensity auto-correlation:

$$
\int I(t) I(t-\tau) \mathrm{d} t
$$

Part of the output of the correlator is sampled by a beam splitter and sent through a polarizer $\mathrm{P}$ into a frequency doubling KDP crystal (second harmonic generation type I). As the delay $\tau$ is scanned, the detector $D_{2}$ records the second harmonic of the successive constructive-destructive interferences of the two arms of the correlator:

$$
\int\left\{[E(t)+E(t-\tau)]^{2}\right\}^{2} \mathrm{~d} t
$$

An example of simultaneous recording on $D_{1}$ and $D_{2}$ is shown at the bottom of the figure. quarter wave plate, before being reflected upon itself. The double passage of each beam through the quarter wave plate will make them successively circularly polarized, thereafter linear, orthogonal to the original direction of polarization, and they will exit the optical system as two mutually orthogonally polarized beams. Second harmonic generation type II in urea will result in a UV signal proportional to the product of the intensities from each arm of the correlator. These measurements are very crude, since not only is all phase information lost, but also all information about the pulse asymmetry (since these auto-correlations are always symmetric). To extract phase information, one has to look at the individual optical cycles, to determine whether or not the light period is constant along the pulse. The pulse itself being the only available probe, that information will have to be extracted from interferences of the pulse with itself. These can be obtained by inserting a polarizer after the correlator in figure 1 , and recording the second harmonic of the successive constructive-destructive interferences as the relative delay between the two arms of the correlator is varied. Although such a recording does indeed carry phase information, it is still not sufficient to lead to a complete signal characterization. It is possible to reconstruct completely the pulse, under the limiting assumption that the pulse is exactly symmetric in amplitude and phase. There is however no physical justification for such an assumption, and thus one additional information is needed to resolve the symmetry of the signal. This information can be provided by the pulse spectrum. We have shown that, indeed, it is possible to reconstruct completely the pulse shape, in amplitude and phase, by iterative fitting of the pulse auto-correlation and spectrum [3]. The method was applied to the determination of the shape and modulation of the pulses emitted by a particular ring dye laser [4]. The accuracy of this method was verified by two different methods. Cross-correlation methods were used [3] when a shorter (compressed) pulse could be generated. In other cases, the propagation through glass of the pulse (for which the phase and modulation had been determined) was computed. Thereafter, the interferometric and intensity autocorrelations of the propagated pulse was computed, and compared to the measurement. The excellent agreement demonstrated the accuracy of the iterative fitting method.

The pulse shape determination by the method detailed in reference [3] is not totally unambiguous. Let us consider for instance a linearly chirped pulse (downchirp) with a steep rise and a longer fall time. The Fourier spectrum of that pulse will have its steeper edge at the low frequency side. It should be noted that the set of three measurements is unchanged if the time axis is reversed (i.e. an upchirped 
pulse with a slow rise and steep decay will match the same set of measurements). This ambiguity can easily be resolved by measuring whether propagation through a thin glass sample broadens or compresses the pulse. In the latter case, the initial modulation corresponded to a downchirp. Another degeneracy occurs in the case of a vanishing phase modulation. In that case, all data (spectrum and auto-correlations) are symmetric, and the pulse shape determination is not unique. The most unattractive feature of that method is that it requires tedious iterations, which can be very lengthy and time consuming, particularly if the pulse has not a simple shape and modulation. We developed therefore another method which yields, in a single measurement, the amplitude and phase of any fs signal [5].

2.2 THE ASYMMETRIC CORRELATOR. - The basic technique is to transform the pulse to be measured by an operation involving a well known transfer function, to a shape that is easily measured. The simplest operation is propagation through glass, which causes the pulse to be broadened by normal dispersion. The broadened pulse is then cross correlated with the original (shorter) pulse. The intensity cross-correlation yields information on the amplitude shape of the signal, while its phase can be extracted from the interferometric cross-correlation. The experimental set-up sketched in figure 2 is similar to the autocorrelator shown in figure 1 , except for the block of glass that has been inserted in one arm of the interferometer. The second harmonics are digitized and stored on a PC. The fasted data acquisition speed enables us to take a set of data every $1.2 \mathrm{~ms}$, and record 30,000 points in a single run. The delay arm or the interferometer is driven by a synchronous clock motor (1/2 turn/minute) coupled to the micrometer of a translation stage (advance of $0.5 \mathrm{~mm} /$ turn). The delay is thus incremented at an average rate of $10 \mathrm{~nm}$ in $1.2 \mathrm{~ms}$. With our instrumentation, we are thus able to record interferometric correlations with a maximum resolution of 60 points/optical period. In order to correct for the speed irregularities of the motor drive and reducing gears, interference fringes of a $\mathrm{He}-\mathrm{Ne}$ laser are recorded, and provide a calibrated delay measurement.

An intensity cross-correlation can be obtained by numerical averaging of the interferometric crosscorrelation. In the experimental set-up of figure 2 , we have chosen to perform the measurement of the intensity cross-correlation independently from that of the interferometric cross-correlation. The redundancy is justified by the high accuracy requirement for the intensity cross-correlation. The reconstruction process outlined below is indeed extremely sensitive to any error on the evaluation of the amplitude shape of the broadened pulse.

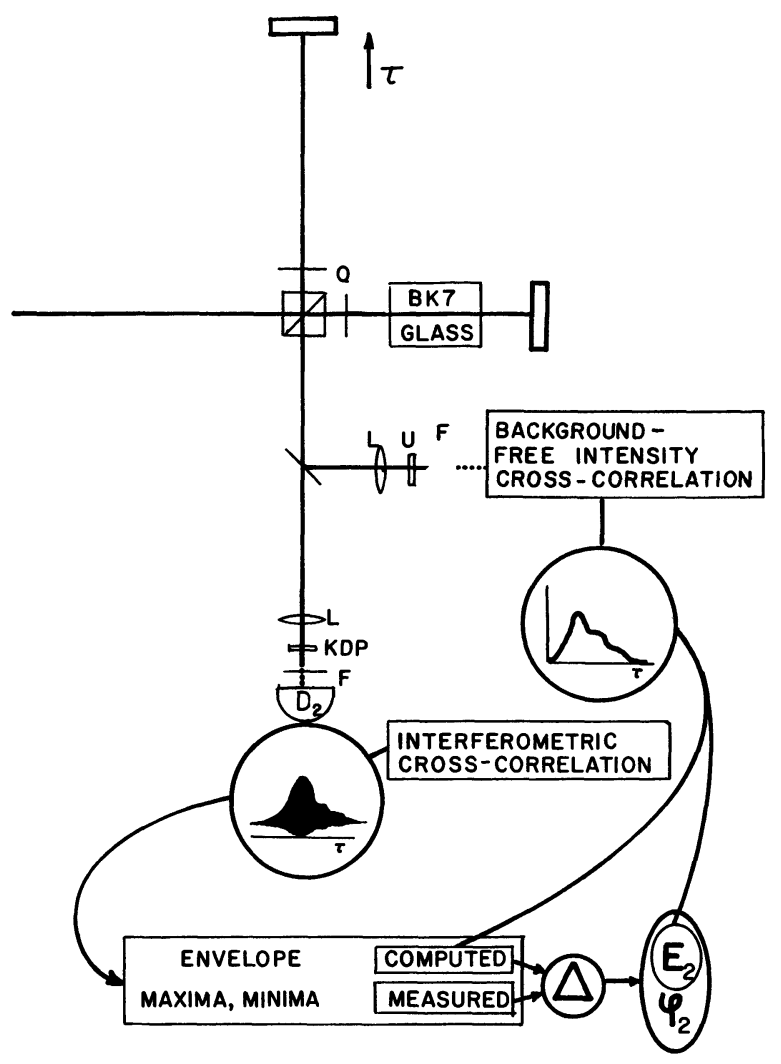

Fig. 2. - Modified correlator for the determination of pulse amplitude and phase. $Q$ : quarter wave plates. $L$ : lenses. U : urea crystal. F : filters (to block the fundamental).

Let $E_{1}(t)$ and $E_{2}(t)$ be the amplitude of the original laser pulse, and that of the pulse broadened by glass. The intensity cross-correlation $A(\tau)$ is by definition :

$$
A(\tau)=\int E_{1}(t-\tau) E_{2}(t) \mathrm{d} t
$$

A rough estimate of $E_{1}$ can be used to compute the Fourier transform $E_{2}(\Omega)$ of the broadened pulse :

$$
E_{2}(\Omega)=\left\{A(\Omega) / E_{1}^{*}(\Omega)\right\}
$$

where $A(\Omega)$ is the Fourier transform of the measured intensity cross-correlation. The amplitudes $E_{1}(t)$ and $E_{2}(t)$ are used to compute the upper and lower envelopes of an interferometric cross-correlation assuming no chirp :

$$
F^{ \pm}(\tau)=\int\left\{E_{1}(t-\tau) \pm E_{2}(t)\right\}^{4} \mathrm{~d} t
$$

The phase $\varphi_{2}(t)$ of the pulse broadened by glass can be determined from the difference $\Delta(\tau)=$ $\left\{F^{+}(\tau)-G^{+}(\tau)\right\}-\left\{F_{-}(\tau)-G_{-}(\tau)\right\}, \quad$ where $G^{+}(\tau)$ and $G_{-}(\tau)$ are the upper and lower envelopes of the measured interferometric cross-cor- 
relation. Indeed, if the pulse $E_{1}$ is much shorter than the pulse $E_{2}$ :

$$
\begin{aligned}
\Delta(\tau)=4 \int E_{1}^{2}(t) & E_{2}^{2}(t-\tau) \times \\
\times & {\left[1-\cos \left\{2 \varphi_{2}(t-\tau)\right\}\right] \mathrm{d} t . }
\end{aligned}
$$

A similar decorrelation procedure as in equation (2) is used to extract $\cos \left(2 \varphi_{2}\right)$ - hence the phase of the broadened pulse $E_{2}$ - from equation (4). This procedure leads thus to a complete determination of the complex electric field amplitude of the pulse broadened by glass. Taking the Fourier transform of this pulse, and multiplying by the transfer function of the glass traversed, leads to the Fourier transform of the original pulse. A last inverse Fourier transform leads to the complex amplitude - hence the amplitude and phase - of the original laser pulse. It should be noted that the procedure outlined above computes the spectrum of the laser pulse, which can be used as an independent test of the method by comparing it to a measured pulse spectrum.

An example of application of this method is shown in figures $3 \mathrm{a}$ and $3 \mathrm{~b}$. The measurements pertain to two different modes of operation of the Antiresonant Ring (ARR) dye laser [6]. This laser exhibits practically indistinguishable intensity auto-correlation when pumped continuously or synchronously (hybrid mode-locking). The propagation of these pulses through dispersive media is drastically different in the two modes of operation [6], and the pulse spectra have opposite asymmetry. Applying our diagnostic technique to the output of the ARR laser, we find that indeed, the frequency modulation of the passively mode-locked laser (Fig. 3a) shows much more structure than that of the synchronously modelocked laser (Fig. 3b). The very strong frequency modulation of the pulses from the ARR laser should be contrasted to the bandwidth limited or linearly chirped output that can be obtained of our ring laser $[3,4]$. The basic difference between the two types of laser can easily be explained by the focusing geometry in the absorber jet. The pulse energy density in the absorber of our ring laser [3] is approximately 6 times the saturation energy density of the DODCI. At that energy density, the dominant contribution to phase modulation is due to off-resonant saturation of the absorber, resulting in a downchirp which can easily be compensated by the normal dispersion of a single intracavity prism [7]. Because much tighter focusing is used in most mode-locked ring lasers [8, 9], the frequency modulation is a complex superposition of that due to Kerr effect and off-resonance saturation of the dye. The focusing in the absorber jet of the passively mode-locked ARR laser [6] is such that the energy density of the pulse is more than one order of magnitude larger than in our ring laser [3]. As a result, the Kerr effect induced chirp becomes of the same order of magnitude as the
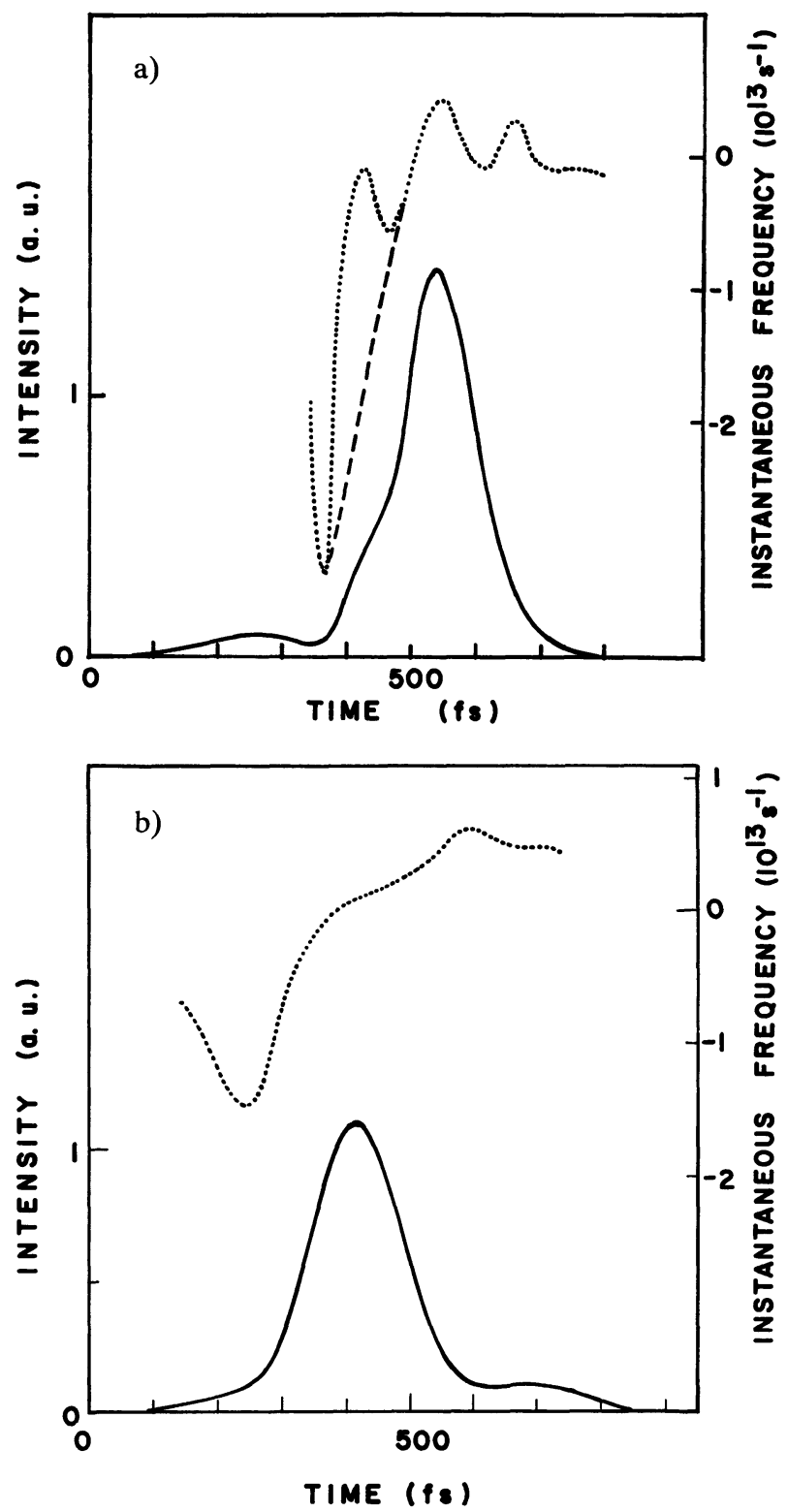

Fig. 3. - Determination of the pulse amplitude and phase using the set-up sketched in figure 2, for the ARR laser. The pulse intensity (solid line) and instantaneous frequency (dotted lines) are plotted as a function of time, for the passive (Fig. 3a) and hybrid (Fig. 3b) mode-locking.

saturation induced chirp, which occurs earlier in the pulse [7]. The contributions of the resonant absorber and that of the Kerr effect (dashed line) can clearly be distinguished in figure $3 \mathrm{a}$. In the case of the synchronously (hybrid) mode-locked ARR laser, the energy density in the absorber is even higher, such that the contribution of the DODCI to the frequency modulation becomes negligible (or too far ahead of the peak of the main pulse). The remaining source of frequency modulation is the Kerr effect in the solvent, which is partly compensated by propagation of the pulses through the cavity with negative dispersion [6], resulting in the chirped pulse shown in figure $3 b$. 


\section{The need for complete pulse characterization.}

The propagation properties of ultrashort pulses are critically dependent on their particular phase modulation. For instance, a significant pulse distorsion is observed for the pulses from the passively modelocked ARR laser after propagation through only $2 \mathrm{~mm}$ of glass, while a similar broadening requires several $\mathrm{cm}$ of glass in the case of our ring laser [3]. In most experiments, there is a particular position where the shortest pulses are desirable, which is generally not at the output mirror of the source. Most often, the pulses will have to traverse media with normal dispersion. When multi-stage dye amplifier chains are used, several $\mathrm{cm}$ of dye solution may contribute to significant pulse broadening. The downchirp characteristic of the ring laser used with moderate focusing in the absorber offers the unique possibility of tayloring the pulses to the dispersion of the amplifier chain. Indeed, by adjusting the amount of intracavity glass, pulses with a controllable linear downchirp are generated [3], thus eliminating the need for a fiber-grating-pair compressor scheme at the end of the chain [10].

Distortion compensation is also an important consideration in time domain reflectometry and optical imaging, an application that is discussed in the next section. An optimum resolution is achieved when the pulse that has propagated through twice the depth of observation has been compressed to its minimum pulse width. A controllable «downchirp » is thus desirable to ensure an optimum spatial resolution throughout the sample. It should be noted that, in this applications, continuous monitoring and correction of the downchirp for maximum depth resolution can be exploited to resolve some of the uncertainties about the optical density of the continuous media being traversed.

The complete characterization of a pulse in amplitude and shape is particularly important in transmission measurements. It offers the possibility to measure directly the time evolution of the complex dielectric constant of a nonlinear medium. There are other applications where the complete knowledge of the pulse amplitude and phase is essential for a correct interpretation of the data. Such is the case for all experiments involving coherent interactions, such as Degenerate Four Wave Mixing (DFWM) [11]. In other experiments such as time resolved fluorescence [12] where the ultimate temporal resolution is sought, knowledge of the pulse fall time or rise time is needed to deconvolute the data.

\section{Three-dimensional imaging.}

The femtosecond temporal resolution that can be achieved with up-conversion detection techniques translate in a spatial resolution or the order of a few microns in range gating applications. This spatial resolution has been exploited in the evaluation of fiber connectors by time domain reflectometry [13]. In the latter application, the weak backscattered reflection from a defect in a fiber is up-converted with a reference pulse in a urea crystal [14]. Not only does this method outperform conventional time domain reflectometers by its resolution (typically $10 \mu \mathrm{m})$, but also by its sensitivity. In preliminary experiments [13] reflection coefficients of $10^{-5}$ were routinely measured. This sensitivity figure has been improved by several orders of magnitude through the use of single photon detection instrumentation [12]. Since the detection is nonlinear (involving the product of a reference pulse by the backscattered signal), it is proportional to the square of the intensity of the laser pulse, hence the detection sensitivity can be further enhanced through amplification of the laser. The ultimate performances of the cross-correlation detection are achieved when the intensity of the reference signal $I_{1}$ is sufficient for a single signal photon to be up-converted in a single UV photon. The expression for $I_{1}$ (Appendix A) indicates a better conversion for smaller numerical apertures into the nonlinear crystal. This conflicts with the requirement of short temporal resolution, which calls for short crystals and tight focusing. We have found empirically that the desired temporal resolution of $10 \mu \mathrm{m}$ can be achieved with a $25 \mathrm{~mm}$ focal distance lens, producing a beam waist of $9 \mu \mathrm{m}$. Under these conditions of focusing, we find that the single photon conversion requires a laser power of $P_{1}=50 \mathrm{~kW}$ (Appendix A), which is only one order of magnitude higher than the peak power of most mode-locked femtosecond dye lasers. The ratio of the available peak laser power to $P_{1}$ is the minimum number of detectable photons/pulse. Unlike standard radar techniques, scanning the probing beam cannot provide a transverse resolution comparable to the $10 \mu \mathrm{m}$ depth resolution, unless complicated tomographic scanning and reconstruction techniques are used [15]. However, up-conversion techniques can provide directly transverse resolution, by detecting the complete (range gated) two-dimensional image in second harmonic. The second harmonic selects not only the depth of illumination, but also the component of the scattered signal exactly parallel to the illuminating beam. The individuality of each point of the scattering surface at a depth $z$ is conserved. It should therefore be possible to record directly an optical image in the up-converted beam. The results of a demonstration experiment are illustrated in figures $4 \mathrm{a}$ and $4 \mathrm{~b}$. The interrogating beam was expanded 10 times and collimated. The « object» was a set of intersecting lines (Fig. 4a), $1 \mathrm{~mm}$ apart, or a « 9 » (1 mm height) drawn on the surface of an aluminum mirror. No signal is observed at the detection, unless the depth of observation is set exactly at the value corresponding to the position 


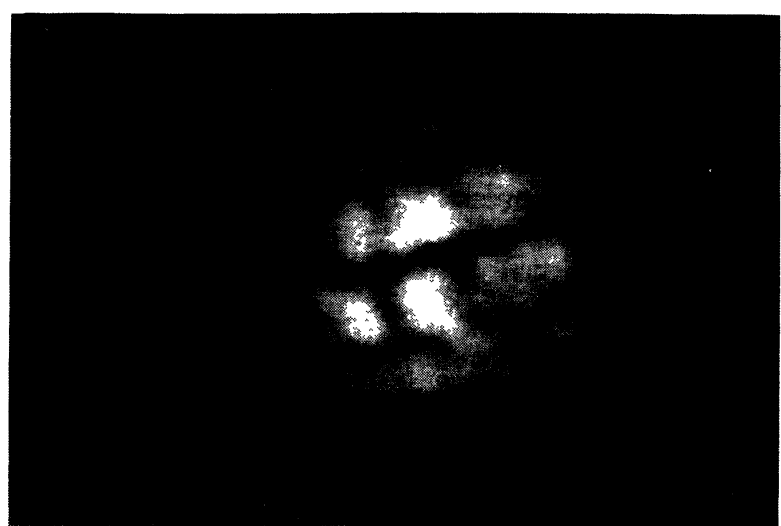

a)

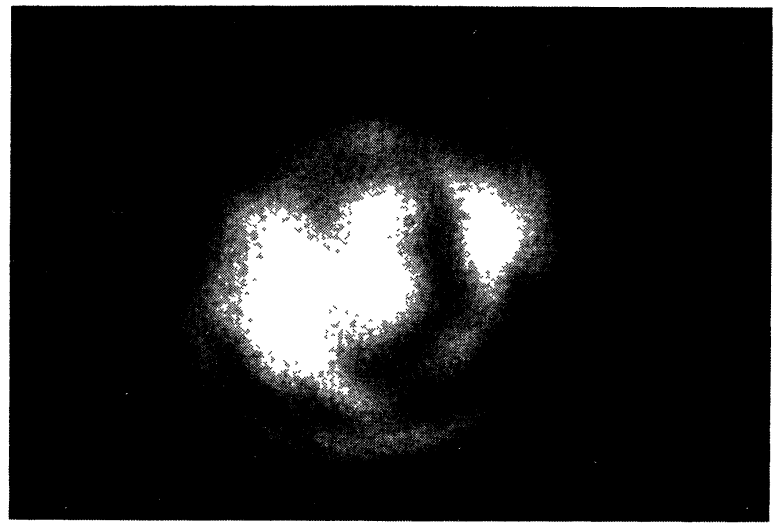

b)

Fig. 4. - Range-gated images recorded in second harmonic. The original images are a set or intersecting lines $1 \mathrm{~mm}$ apart (Fig. $4 \mathrm{a})$, or a small « $9 »(1 \mathrm{~mm}$ height $)$ drawn on an aluminum mirror.

of the sample. For the correct setting of the depth parameter, the detection records the pictures shown in figure 4 . The relative blurr of the picture is partly due to the large magnification factor, and partly to the difficulty of focusing the invisible UV image onto a photographic plate. Real time adjustments of the optical system with a UV vidicon camera would solve this technical difficulty.

\section{Acknowledgment.}

This work was supported by the National Science Foundation, under Grant No. ECS-8406985.

\section{Appendix A.}

Laser power for optimum sensitivity.

The second harmonic field $E_{2}$ generated by frequency doubling of a field $E_{1}$ over a distance $L$ (assuming perfect phase matching) is given by :

$$
E_{2}(L)=E_{1}(0) \tanh \left\{L / L_{\mathrm{sh}}\right\}
$$

where the characteristic distance $L_{\mathrm{sh}}$ is given by [16]

$$
L_{\mathrm{sh}}^{-1}=\frac{4 \pi \omega^{2} d}{k_{1} c^{2}} E_{1}(0) .
$$

In equation (A.2), $k_{1}$ is the wave vector of the light, $c$ the speed of light in the medium, $\omega$ the (fundamental) light frequency, $d$ the nonlinear coefficient (cgs units). For practical purpose, we consider that a crystal of twice the characteristic length will provide full conversion. For a given crystal length $L$, the condition $L_{\mathrm{sh}}=L / 2$ implies a light intensity of

$$
I_{0}=0.019 \frac{1}{n}\left\{\frac{\lambda}{L d}\right\}^{2}
$$

where $I_{0}$ is in $\mathrm{W} / \mathrm{cm}^{2}, L, \lambda$ and $d$ in cgs ; $n$ is the index of refraction of the crystal. The reference intensity required to convert a single signal photon into a single second harmonic photon is twice the intensity given by (A.3). The useful crystal length is of the order of the confocal parameter $\rho=w_{0} / \theta$. After substitution in equation (A.3), we find the reference intensity required for «single photon upconversion » to be :

$$
I_{\text {ref }}=0.038 \pi^{2} \theta^{4} / n^{3} d^{2}
$$

the nonlinear coefficient of urea is $2.8 \times 10^{-9} \mathrm{cgs}$, and the index of refraction 1.48. The smallest $\theta$ allowable to maintain a temporal resolution better than $100 \mathrm{fsec}$ is taken to be 0.015 . With a spot size given by $w_{0}=\lambda /(\pi \theta)$, in equation (A.4) yields for the pulse power :

$$
P_{\text {ref }}=I_{\text {ref }} \pi \frac{w}{2}=\theta^{2} \times 9 \times 10^{6} \mathrm{~W} .
$$

Focusing a beam of $0.7 \mathrm{~mm}$ diameter with a $25 \mathrm{~mm}$ focal distance lens corresponds to a $\theta$ of 0.015 . In this geometry, the laser power required for $100 \%$ conversion is $2 \mathrm{~kW}$.

\section{References}

[1] SCHON, K., «An iterative deconvolution algorithm for the reconstruction of high-voltage impulses distorted by the measuring system » in Fast electrical and optical measurements, Eds J. E. Thompson and L. H. Luessen (Martinus Nijhoff Publishers, Dordrecht) 1986.
[2] SchON, K., GiTt, W., «Reconstruction of high impulse voltages considering the step response of the measuring system »IEEE Trans. Power Appar. Syst. 101 (1982) 4147-4155.

[3] Diels, J.-C., Fontaine, J. J., McMichael, I. C. and SimONI, F., «Control and measurement of 
ultrashort pulse shapes (in amplitude and phase) with femtosecond accuracy " Appl. Opt. 24 (1985) 1270-1282.

[4] Dietel, W., Fontaine, J. J. and Diels, J.-C., «Intracavity pulse compression with glass : a new method of generating pulses shorter than 60 femtoseconds » Opt. Lett. 8 (1983) 4-6.

[5] Diels, J.-C., Fontaine, J. J., Jamasbi, N., Ming LAI and MACKEY, J., The Femto-nitpicker, CLEO 87, Baltimore (May 1987).

[6] Diels, J.-C., Jamasbi, N. and Sarger, L., « Passive and hybrid femtosecond operation of a linear astigmatism compensated dye laser " Ultrafast Phenomena $V$, Eds G. R. Fleming and A. E. Siegman (Springer Berlin) 1987, pp. 2-4.

[7] Diels, J.-C., Dietel, W., Fontaine, J. J., RudOLPH, W. and WilHeLMI, B., « Analysis of a mode-locked ring laser : chirped-solitary-pulse solutions » J. Opt. Soc. Am. B 2 (1985) 680-686.

[8] Fork, R. L., Green, B. I. and Shank, C. V., "Generation of optical pulses shorter than 0.1 ps by colliding pulse mode-locking " Appl. Phys. Lett. 36 (1981) 671-672 ;

VAldAMIS, J. A., Fork, R. L., Gordon, J. P., Opt. Lett. 10 (1985) 131.
[9] Yamashita, M., IshikaWA, M., TORIZUKA, K. and SATo, T., «Femtosecond pulse laser chirp compensated by cavity-mirror dispersion »Opt. Lett. 11 (1986) 504-506.

[10] Nikolaus, B. and GrischKovsky, D., « 12x Pulse compression using optical fibers »Appl. Phys. Lett. 42 (1983) 1.

[11] Diels; J.-C. and McMichael, I. C., « Degenerate four-wave mixing of femtosecond pulses in an absorbing dye jet »J. Opt. Soc. Am. B 3 (1986) 535-543.

[12] Rudolph, W. and Diels, J.-C., " Femtosecond resolved fluorescence "Ultrafast Phenomena $V$ (Springer Berlin) 1986, pp. 71-74.

[13] Fontaine, J. J., Diels, J.-C., Wang, C. Y. and SallabA, H., «Subpicosecond time domain reflectometry » Opt. Lett. 6 (1981) 495-498.

[14] DiElS, J.-C. and WANG, C. Y., « Accurate range gating with mode-locked dye lasers » SPIE meeting on picosecond lasers and applications, Proc. Soc. Photo. Opt. Instrum. Eng. 322 (1982) 166.

[15] Diels, J.-C. and FonTAine, J. J., « Imaging with short optical pulses » Opt. Lasers Eng. 4 (1983) 145-165.

[16] Zernike, F. and Midwinter, J. E., Applied Nonlinear Optics, John Wiley (New York) 1973, p. 48. 